\title{
Gold Contact Resistance
}

\section{A MICROPROCESSOR-CONTROLLED MEASUREMENT SYSTEM}

\author{
H. Becker and R. Schnabl
}

W.C. Heraeus GmbH, Hanau, Federal Republic of Germany

\begin{abstract}
Due to their excellent electrical, chemical and mechanical properties gold and gold alloys are well proven materials for electrical applications related to the switching and transmission of low voltage signals. In particular, high performance electrical contacts either consist of gold or are finished with gold or gold alloys $(1,2)$. These contacts are expected to bave a low and stable contact resistance, even under severe environmental conditions, for periods of service of up to 30 years. $A$ quick and reliable method of measuring the contact resistance of surfaces is of great value for quality control in the production of devices.
\end{abstract}

This article describes the principles behind, and some test results obtained when using, an improved contact resistance measurement system. This has been developed for the quality control and assurance of gold and other precious metal layers, and manufactured contact devices (3). The compact microprocessor-controlled equipment performs in a relatively short time a large number of sensitive and reproducible measurements on specimens of various shapes. The application of the equipment has been demonstrated by contact resistance measurements on electroplated gold layers of purity between 16 and 24 carat.

A universal contact resistance measurement system should fulfil a number of requirements. For instance, the instrument should be suitable for both laboratory tests and routine measurements, and all the principal parameters (contact

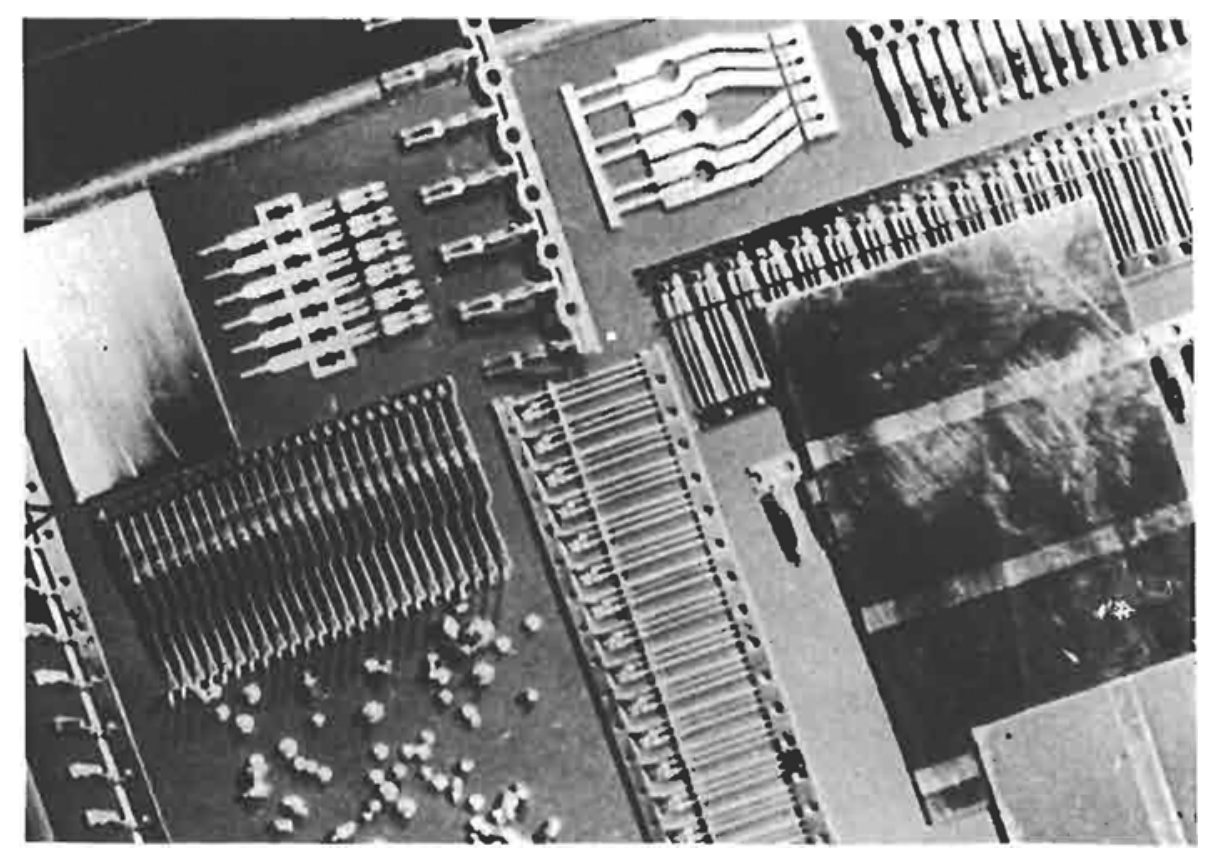

force, contact impulse, time to stable readings, friction path length) should not only be variable over a wide range, but also be capable of reproducible adjustment. The reference electrode should be designed not only for use with rivets and wires such as those specified by ASTM B667, but also with other forms of reference contacts, for example of the rotating type. In order to prevent the spread of impurities from one contact point to the next, a fresh probe surface should always be available insofar as the geometry of the contact permits, and a statistical evaluation of the results should be possible.

Standard electrical measurement conditions, that is 'dry circuit' (4), must be observed, and the equipment should be capable of being operated at need by personnel who are not highly trained. Any adjustments that may be needed during measurements should be simple ones. Lastly, such an instrument should be readily adaptable to the widest possible range of test specimen forms (rivets, spring contacts, pins and blades) and starting materials (band, strip, wire, profiles) and special forms, such as semiconductor substrates. Examples of the many types of contacts available with selectively plated precious metal layers which are potential test specimens are shown in Figure 1.

Fig. 1 Examples of selectively gold plated connectors and contacts of various configurations, which may be tested with the method discussed in this article 
Fig. 2. The KWA 07 contact resistance measurement equipment with some of its main features indicated:

1 Detector unit which interfaces with the microprocessor and the power unit driving the sensor lift and specimen stepping motors

2 Interchangeable reference electrode holder

3 Specimen stage with sample magazine

4 Magazine with samples

5 Microprocessor capable of handling 8 bits in parallel, with input keyboard

6 Digital voltmeter

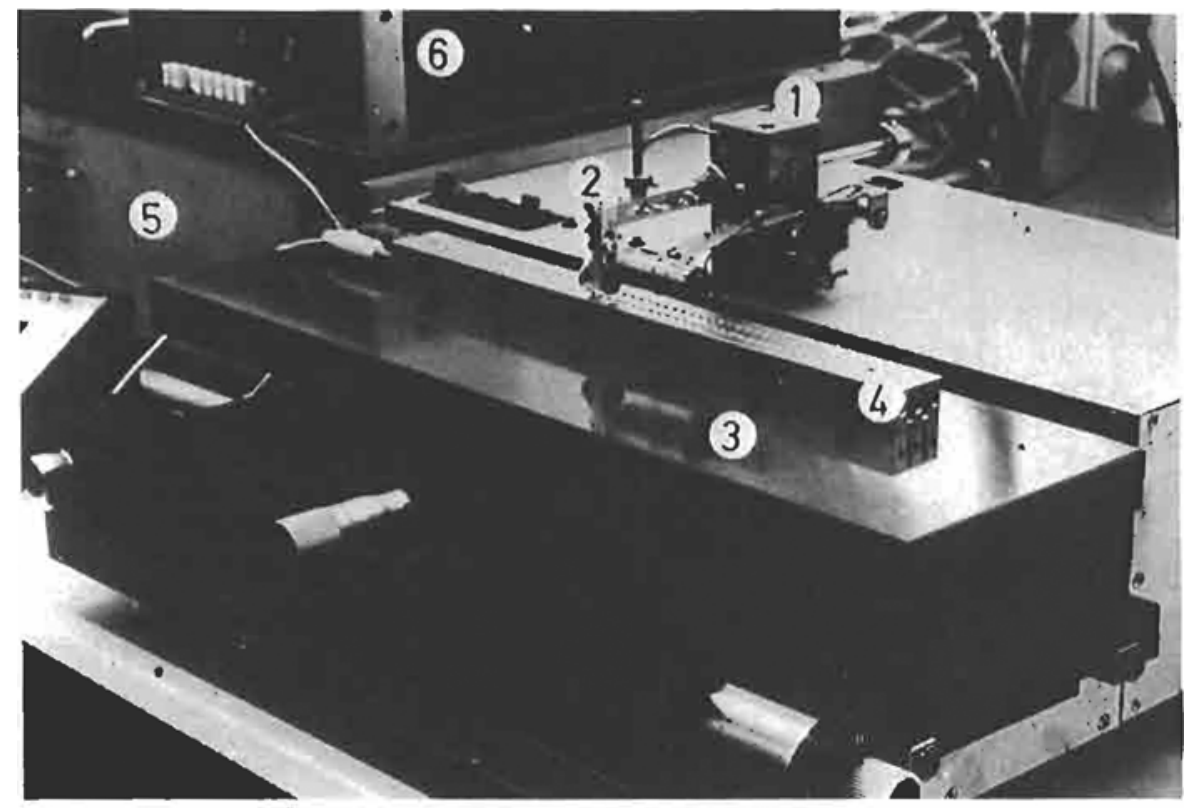

It is believed that the new contact resistance measurement system used in this investigation fulfils the test requirements described above. It also incorporates a microprocessor control unit which enables complex specimen shapes to be positioned without restriction relative to the reference electrode. A data processing system complete with printout is also included. The complete contact resistance measurement system is shown in Figure 2.

\section{Application to Plated Contacts}

The new contact resistance measurement technique has been shown to be sensitive to the composition and type of gold deposit on plated contact surfaces when tests were carried out on four different gold electrodeposits. These were:

(1) 24 carat gold deposited at $0.5 \mathrm{~A} / \mathrm{dm}^{2}$ from a neutral cyanide electrolyte

(2) 23.5 carat gold-cobalt deposited at $1.0 \mathrm{~A} / \mathrm{dm}^{2}$ from an acid cyanide bath

(3) 23 carat gold-nickel deposited at $5.0 \mathrm{~A} / \mathrm{dm}^{2}$ from an acid cyanide solution

(4) 16 carat gold-copper-cadmium deposited at $2.8 \mathrm{~A} / \mathrm{dm}^{2}$ from a mildly alkaline cyanide electrolyte.

The thickness of each deposit was $2 \mu \mathrm{m}$ and a contact force of $0.05 \mathrm{~N}$ was applied through a fine gold probe in all the tests. The general measurement procedure and special features of the method have been described previously by the authors (3).

The dependence of the contact resistance of gold alloy surfaces on the alloy composition is indicated by the equation proposed by Holm (5) to describe the relationship between contact resistance, component resistivities and contact force:

$$
\mathrm{R}_{\mathrm{C}}=\frac{\rho_{1}+\rho_{2}}{2}\left(\frac{\mathrm{H}}{\mathrm{F}}\right)^{1 / 2}
$$

where

$\mathrm{R}_{\mathrm{C}}$ is the contact resistance

$\rho_{1}$ and $\rho_{2}$ are the electrical resistivities of the two contacting components - in this case the plated gold alloy and the pure gold probe

$\mathrm{H}$ is the hardness of the softer material - the gold probe in this experiment

$F$ is the contact force.

It can be appreciated that, if $\mathrm{H}, \mathrm{F}$ and $\rho_{2}$ are held constant, as was the case in the present investigation, for a given test environment the measured contact resistances depend upon the electrical resistivities of the different electroplated gold alloys in the as-deposited states, which in turn are related to compositions.

Statistical analysis of the contact resistance data has shown a clear dependence of this variable on the composition of the gold deposit (Figure 3). The distribution curves shown in this figure indicate that 90 per cent of the measurements of contact resistance of electrodeposited pure gold, gold-cobalt, gold-nickel and gold-copper-cadmium are less than 2, 7, 22 and 200 milli-ohms, respectively. The steep slopes of the distribution curves obtained confirm generally good reproducibility within the tests. The 16 carat gold-coppercadmium alloy deposit, however, exhibited a wide range of contact resistances, with values from about 20 to 700 milli- 


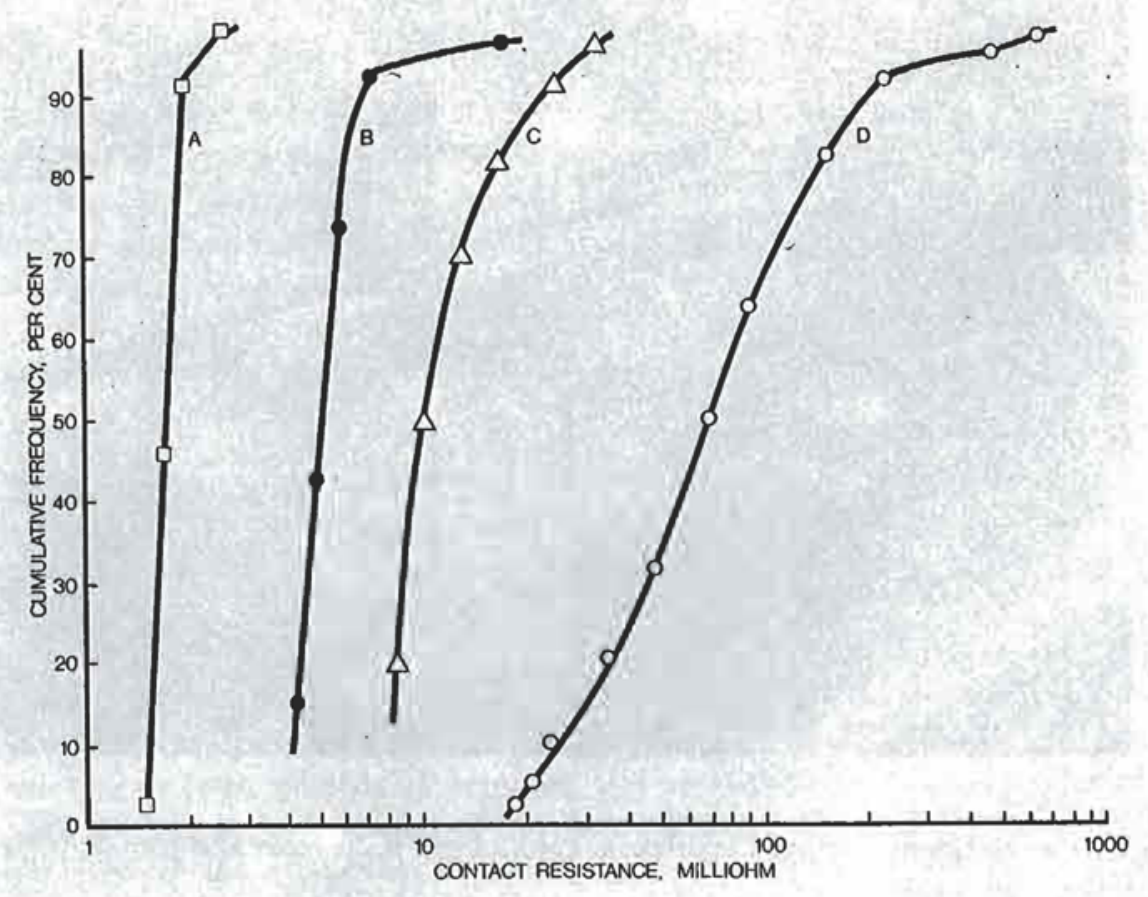

Fig. 3 Cumulative frequencies of contact resistance values obtained on four types of electrodeposited gold surfaces, using a contact force of $0.05 \mathrm{~N}$ with a pure gold probe:

A 24 carat gold

B 23.5 carat gold-cobal

C 23 carat gold-nickel

D 16 carat gold-copper-cadmium ohms under the test conditions, which implies that this alloy should be included in contact applications only after very careful design considerations.

\section{Conclusion}

The measurement of contact resistance with a reference electrode is a proven method of obtaining information regarding the surface cleanliness of gold and other metals. It has been found that refinements in the technique reported here have improved the sensitivity of the equipment, and reproducibility of the measurements, to the extent that real differences in contact resistances obtained for gold alloy deposits of varying purities and compositions are clearly revealed.

\section{References}

1 W. Reyes, R. Currence, E.F. St. Peter, J. Liao and G. Bolger, in 'Proceedings of the 13th. Annual Connector Symposium', Philadelphia, Oct. 8 and 9,1980 , pp. $1-10$

2 G.J. Russ, R.J. Chesseri, in 'Proceedings of the 24th Annual Holm Conference on Electrical Contacts', Chicago, II., 1978, pp. 227-233

3 H. Becker and R. Schnabl, Metalloberfläche, 1980, 34, (12), 510-515

4 DIN 4160

5 R. Holm, 'Electrical Contacts Handbook', Springer Verlag, Berlin, Göttingen, Heidelberg, 1958 